Original article

\title{
Morphological and numerical assessment of intracranial aneurysms ruptures risk
}

\author{
Dmitriy V. Ivanov, Alexandr V. Dol \\ Saratov State University, Saratov, Russia
}

Received 25 January 2018, Revised 13 March 2018, Accepted 24 April 2018

(C) 2018, Ivanov D.V., Dol A.V.

(C) 2018, Russian Open Medical Journal

Abstract: Background - Rupture of intracranial aneurysms leads to subarachnoid hemorrhage and, as a consequence, to hemorrhagic stroke. Mortality at the same time reaches $45 \%$. Moreover, half of survivors experience serious neurological problems. Diagnosis of cerebral aneurysms is difficult due to the fact that they usually do not have any typical symptoms. Development of criteria for assessing risk of aneurysm rupture is an actual scientific problem, since preventive surgical treatment entails serious post-operative complications and untimely treatment leads to aneurysm rupture. It is necessary to understand whether this or that aneurysm is prone to rupture in order to form a treatment tactic.

Methods - Factors such as size of aneurysm, size ratio, irregular shape and wall shear stress were highlighted in this work. Each of these factors was considered for four models of aneurysms and numerical simulations of the blood flow in these aneurysms were performed. Size ratio was calculated for each model: 1.2, 1.6, 2.0 and 2.5. Height of aneurysms was 3.5, 4.5, 6.0 and 7.5 mm. Aneurysms with size ratios of 2.0 and 2.5 had an irregular shape. Given the morphological factors, 1.2-aneurysm and 1.6-aneurysm had small risk of rupture and other aneurysms had high rupture risk.

Results - Numerical biomechanical modeling showed that there were no vortices in small aneurysm during the entire cardiac cycle. Average wall shear stress of aneurysms with 2.0 and 2.5 size ratios was more than two times lower than wall shear stress of the small aneurysm. Wall shear stress values on most of the dome of the largest aneurysm did not exceed $1.5 \mathrm{~Pa}$. The increase in size ratio led to an increase of blood pressure in aneurysm during the entire cardiac cycle. Distribution of wall shear stress in aneurysms with size ratios more than 2.0 turned out to be essentially inhomogeneous. Also these aneurysms had complex flow patterns. Presence of vortices, inhomogeneous distribution of wall shear stress indicates a higher risk rupture of such aneurysms.

Conclusion - It can be concluded that both morphological and hemodynamic factors do not contradict each other, but complement each other. This fact suggests that one can develop objective and convenient for use in preoperative planning of surgical treatment methods for assessing the risk of cerebral aneurysms rupture for a particular patient.

Keywords: intracranial aneurysm, risk rupture, size ratio, irregular shape, size of aneurysm, blood pressure, wall shear stress.

Cite as Ivanov DV, Dol AV. Morphological and numerical assessment of intracranial aneurysms ruptures risk. Russian Open Medical Journal $2018 ; 7:$ e0304.

Correspondence to Dmitriy Ivanov. Address: Department of Mathematical Theory of Elasticity and Biomechanics, Saratov State University, Astrakhanskaya str., 83, Saratov, 410012, Russia. Email: ivanovdv@gmail.com.

\section{Introduction}

Intracranial aneurysms are pathological widenings of cerebral arteries which occur in approximately 2-5\% [1] of the population. Diagnosis of aneurysms is difficult due to the lack of typical symptoms. However, the development of tomographic methods in recent years has significantly increased the likelihood that an aneurysm will be detected in time [2].

Rupture of aneurysms leads to subarachnoid hemorrhage, which is one of the causes of hemorrhagic stroke. Mortality with subarachnoid hemorrhage is up to $45 \%$, and half of survivors have serious neurological problems [3]. Taking into account the high mortality rate, the issue of the need for carrying out preventive surgical or endovascular treatment of an unruptured aneurysm in each case remains open, as it can be associated with serious postoperative complications [4]. Therefore, the problem of developing reliable and objective criteria to assess the risk of rupture is relevant. Moreover, such criteria should work regardless of gender, age, race and other individual parameters of each patient.

For several decades, the authors [5-8] are trying to identify morphological and mechanical factors which determine risk of cerebral aneurysms rupture. Among such factors, first of all, researchers consider size of aneurysms [6]. Authors also estimate size ratio of aneurysm $[1,2,9,10]$, aspect ratio, as well as irregular shape $[4,11]$, wall shear stress [2], effective stresses in the wall, blood pressure in aneurysm dome [12].

Aim of this paper is to show that an aneurysm having a greater risk of rupture in terms of morphological factors is also more prone to rupture in terms of mechanical factors. The study was carried out using methods of computational fluid dynamics (CFD), which makes it possible to evaluate blood flow in aneurysms. 


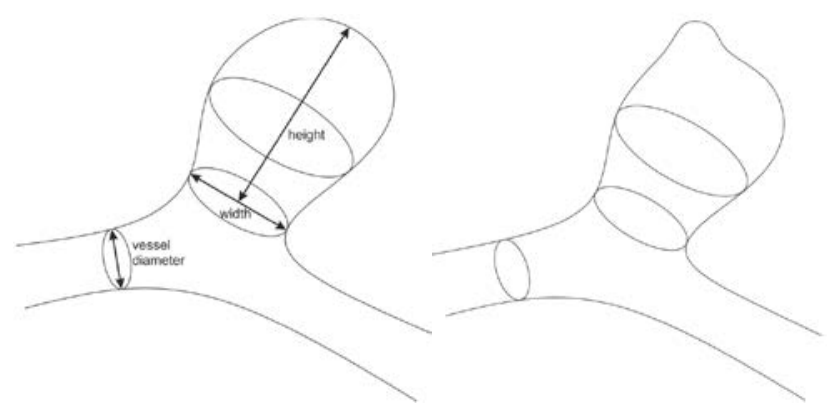

Figure 1. Schematic images of a brain vessel with an aneurysm: the vessel diameter, the height of an aneurysm (width) and the diameter of an aneurysm neck (width) are marked on the left, an irregular shape of aneurysm is shown on the right.

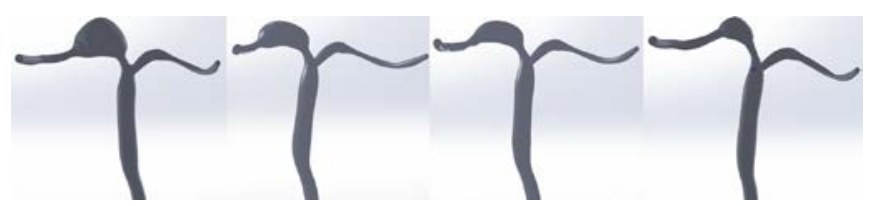

Figure 2. Aneurysm models which were considered in this research (from right to left: 2.5-aneurysm, 2.0-aneurysm, 1.6-anerusym, 1.2-aneurysm).

Table 1. Criteria values of morphological rupture factors

\begin{tabular}{|c|c|c|c|}
\hline Refs & Year & Criterion & Value \\
\hline [12] & 1992 & Size of aneurysm & $\begin{array}{l}4 \mathrm{~mm} \text { or less - for unruptured } \\
5 \mathrm{~mm} \text { and more - for ruptured }\end{array}$ \\
\hline [29] & 2003 & Size of aneurysm & $7 \mathrm{~mm}$ and more - for ruptured \\
\hline [8] & 2008 & Size ratio & 2.05 and more - for ruptured \\
\hline [23] & 2010 & Size ratio & $\begin{array}{l}2.57 \text { - average for unruptured } \\
4.08 \text { - average for ruptured } \\
\text { more than } 3 \text { - for ruptured }\end{array}$ \\
\hline [21] & 2014 & $\begin{array}{l}\text { Size of aneurysm, } \\
\text { irregular shape }\end{array}$ & $9 \mathrm{~mm}$ and more - for ruptured \\
\hline [5] & 2015 & Size ratio & $\begin{array}{l}1.55 \text { - average for unruptured } \\
2.09 \text { - average for ruptured }\end{array}$ \\
\hline [11] & 2016 & Irregular shape & Not availible \\
\hline [18] & 2016 & Irregular shape & Not availible \\
\hline [27] & 2016 & Irregular shape & Not availible \\
\hline [14] & 2016 & Size ratio & $\begin{array}{l}1.07 \text { - average for unruptured } \\
1.55 \text { - average for ruptured }\end{array}$ \\
\hline [20] & 2017 & Size of aneurysm & $7 \mathrm{~mm}$ and more - for ruptured \\
\hline [10] & 2017 & Size of aneurysm & $\begin{array}{l}\text { between } 5 \text { and } 10 \mathrm{~mm} \text { - size of } \\
\text { ruptured }\end{array}$ \\
\hline [25] & 2017 & Size of aneurysm & Not availible \\
\hline [3] & 2017 & $\begin{array}{l}\text { Size of aneurysm, } \\
\text { irregular shape }\end{array}$ & $\begin{array}{l}2.5 \mathrm{~mm} \text { - average for unruptured } \\
6.9 \mathrm{~mm} \text { - average for ruptured }\end{array}$ \\
\hline [9] & 2017 & Size ratio & 2.1 and more - for ruptured \\
\hline [4] & 2018 & Size ratio & Not availible \\
\hline [24] & 2016 & $\begin{array}{l}\text { Size ratio, size of } \\
\text { aneurysm }\end{array}$ & $\begin{array}{l}\text { Size ratio more than } 1.5 \text { - for } \\
\text { ruptured }\end{array}$ \\
\hline [2] & 2013 & Size of aneurysm & $\begin{array}{l}\text { Aneurysms of the anterior } \\
\text { communicating artery with } 4 \mathrm{~mm} \text { in } \\
\text { diameter should be treated. Other } \\
\text { aneurysms less than } 7 \mathrm{~mm} \text { in size } \\
\text { have a small risk of rupture. }\end{array}$ \\
\hline [26] & 2009 & Size ratio & 2.05 and more - for ruptured \\
\hline
\end{tabular}

Table 2. Hemodynamic (mechanical) factors of aneurysm rupture

\begin{tabular}{cccc}
\hline Refs & Year & Criterion & Investigation method \\
\hline$[15]$ & 2015 & Low WSS & Numerical simulation \\
{$[16]$} & 2017 & Low WSS & Numerical simulation, experiments \\
{$[17]$} & 2012 & Low WSS & Numerical simulation, experiments \\
\hline
\end{tabular}

\section{Material and Methods}

\section{Literature review: factors and criteria of intracranial aneurysm rupture}

Size of aneurysm, size ratio, irregular shape and wall shear stress were identified as rupture factors on the basis of literature review. Each of these factors was considered in relation to four models of aneurysms (hereinafter we will title them as 1.2aneurysm, 1.6-aneurysm, 2.0-aneurysm and 2.5-aneurysm) for which numerical calculations of the blood flow were performed.

Further, we present the results of the literature review. First of all, we make some explanations. On the left in Figure 1, an aneurysm with a smooth surface is schematically shown. Vessel diameter, aneurysm height and diameter of the aneurysm neck (width) are marked. There is an aneurysm with irregular shape on the right in Figure 1. Small aneurysm or bleb is present on its dome. Relation to the size of the vessel (size ratio) will be calculated as the ratio of aneurysm height to the vessel diameter.

Next, we present a table with morphological criteria (factors) for the rupture of intracranial aneurysms considered in this paper and their numerical values taken from literature (Table 1).

Along with morphological, the hemodynamic (mechanical) factor was also identified, indicating risk of aneurysm rupture. This factor is low wall shear stress (WSS). Among the articles considered in the framework of the review, following factors were identified and are listed in Table 2.

\section{D models of aneurysms}

Four aneurysms of the basilar artery bifurcation were considered, the models of which are shown in Figure 2. For all models, size ratio was calculated: 1.2, 1.6, 2.0 and 2.5. Dimensions (height) were 3.5, 4.5, 6.0 and $7.5 \mathrm{~mm}$ respectively. Two of four considered aneurysms also had irregular shape; one of them is shown in Figure 3. According to the analysis of literature, the two largest aneurysms should be assigned to a group with a high risk of rupture in such parameters as size of aneurysm, size ratio and irregular shape. On the right in Figure 3, arrow shows bleb on the aneurysm dome.

\section{CFD modeling}

Two models of aneurysms considered in this work have already been analyzed [12], but in that research only their absolute sizes were taken into account in the analysis of the results, and modeling was carried out taking into account elasticity of vessel walls. Simulation results were compared with calculations of healthy vessels and vessels with stenosis. In this paper we introduce into consideration other rupture factors, the applicability of which is shown in recent investigations.

In this research, simulation was carried out in ANSYS CFX 18.0. Blood was considered to be a viscous incompressible homogeneous and Newtonian fluid with constant coefficient of dynamic viscosity $0.004 \mathrm{~Pa}^{*} \mathrm{~s}$ and density $1050 \mathrm{~kg} / \mathrm{m}^{3}$ [12]. Flow was considered as laminar. A nonstationary system of 3D NavierStokes equations was solved. In each artery, including an aneurysm, blood flow parameters such as velocity and pressure at each time of the cardiac cycle were calculated. Calculation was carried out taking into account the boundary conditions at the inlet, outlet and on the side walls of the vessels. Thus, the equations and boundary conditions determined the behavior of the vessels throughout the entire cardiac cycle. 


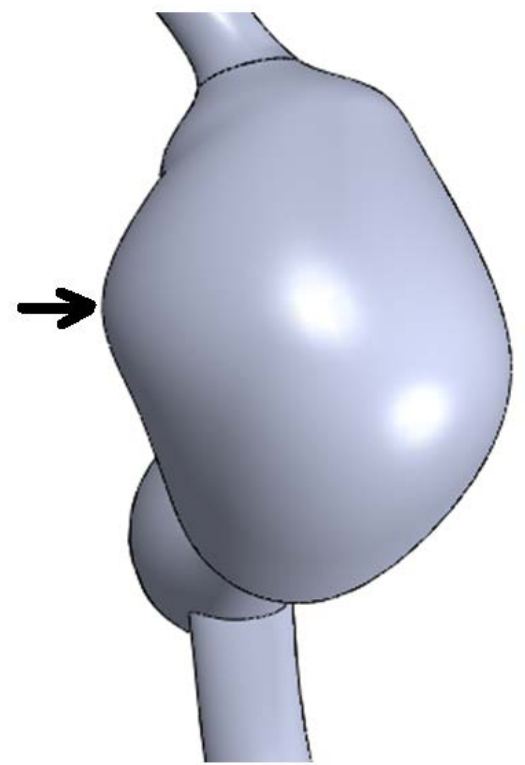

Figure 3. Top view of the largest aneurysm (right) with irregularity of its shape

Inlet velocity, $\mathrm{m} / \mathrm{s}$

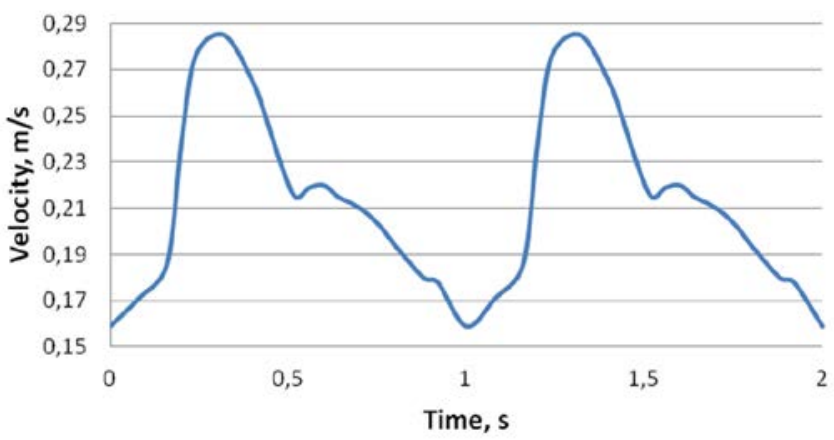

Figure 4. Velocity on basilar artery inlet

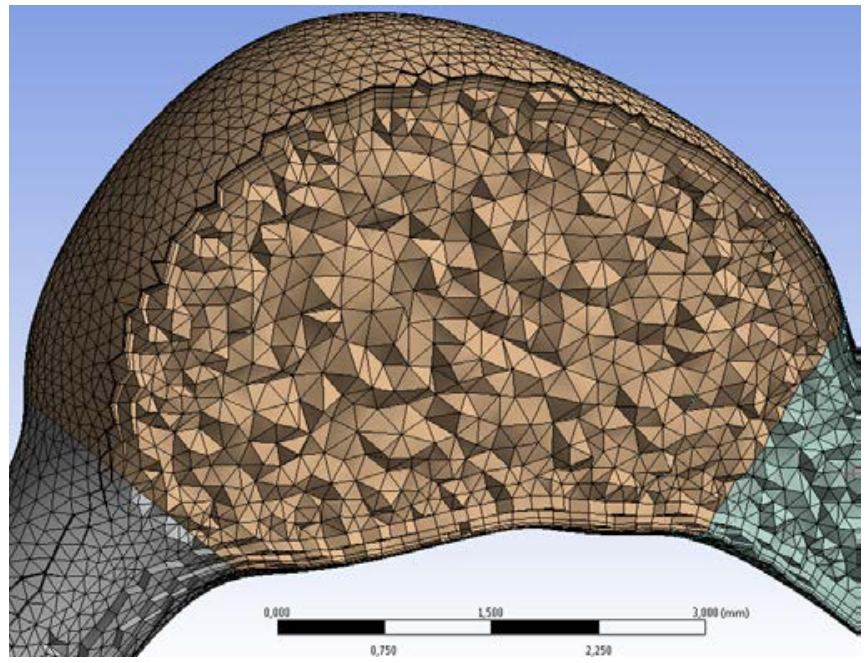

Figure 5. Fragment of tetrahedral computational mesh with prism layers
Table 3. Average WSS and pressure values in aneurysm dome during systole and diastole

\begin{tabular}{lcccc}
\hline Aneurysms & \multicolumn{2}{c}{ Average WSS, $P a$} & \multicolumn{2}{c}{ Average pressure, $P a$} \\
& systole & diastole & systole & diastole \\
\hline 1.2-aneurysm & 5,2 & 3,6 & 816 & 600 \\
1.6-aneurysm & 3.6 & 2.7 & 835 & 625 \\
2.0-aneurysm & 2.6 & 2.0 & 876 & 650 \\
2.5-aneurysm & 2,4 & 1,6 & 913 & 670 \\
\hline
\end{tabular}

WSS, wall shear stress.

Because the lack of information about vessel mechanical properties, the wall elasticity was neglected, and rigid wall boundaries with no-slip conditions were used at the vessel walls. Such assumption is acceptable for the analysis because the influence of wall compliance on the aneurismal flow pattern is insignificant compared with that of overall morphology [14].

At the inlet of basilar artery, time-varying blood velocity was set (Figure 4) [12], at the outlets of posterior cerebral arteries zero pressure boundary conditions were set. Pulse rate was assumed to be 60 beats per minute, blood pressure was considered to be normal.

The problem was solved for two cardiac cycles. Results of the second cycle were analyzed to exclude the effect of zero initial conditions on the solution. Time step was euqal to $0.025 \mathrm{~s}$.

Tetrahedral computational mesh with 6 prismatic layers was constructed. Mesh convergence analysis was performed. Average size of tetrahedron edge was $0.1 \mathrm{~mm}$. Error value was lower than $3 \%$. Fragment of 3D computational mesh is shown in Figure 5.

Each finite-element model contained about 1,000,000 finite elements and about 350,000 nodes. Linear finite elements were used.

\section{Results}

Fields of wall shear stress and pressure on aneurysm dome were analyzed for systole and diastole. Average values of WSS and blood pressure on the dome were placed in Table 3.

With increasing size ratio, pressure in aneurysm dome also increases. Average values of WSS in the dome, on the contrary, decrease substantially in magnitude. In both systole and diastole, mean values of WSS of 2.5-aneurysm differ from those for 1.2aneurysm more than twice. For pressure, situation is similar, but difference here is about $12 \%$.

Now we will show figures with WSS, pressure fields and streamlines only in 1.2-aneurysm and 2.5-aneurysm. Pressure, WSS fields and streamlines in 1.6- and 2.0-aneurysms are similar to the same fields for 1.2- and 2.5-aneurysms, respectively.

Fields of WSS and pressure in 1.2- and 2.5-aneurysms at systole are shown in Figures 6-8.

WSS of 1.2-aneurysm at systole are shown in Figure 6. WSS field of 2.5-aneurysm is shown in Figure 7. From a comparison of Figures 6 and 7 it is evident that WSS field in the dome of 2.5aneurysm is less homogeneous in comparison with the distribution of WSS in the dome of 1.2-aneurysm.

Pressure field on 1.2-aneurysm wall is shown on the left in Figure 8. Pressure field on 2.5-aneurysm wall is shown on the right in Figure 8.

The highest values of blood pressure are concentrated in the upper part of the dome and correspond to the region of the impact of the blood flow entering the aneurysm from the basilar artery. 

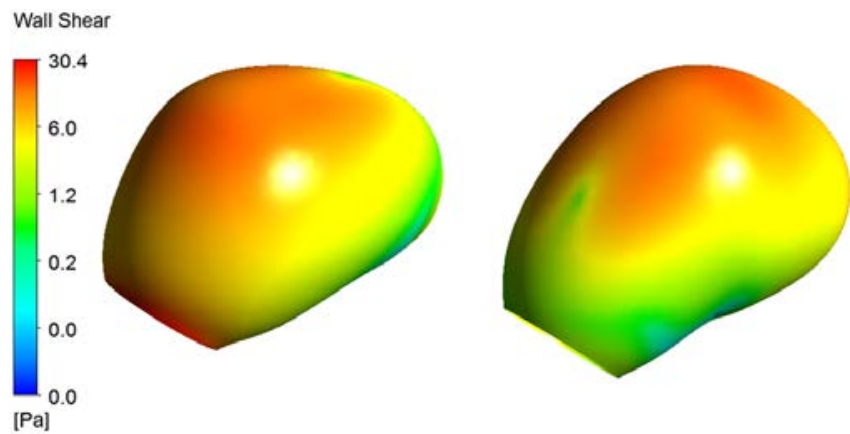

Figure 6. WSS field of 1.2-aneurysm at systole

Wall Shear

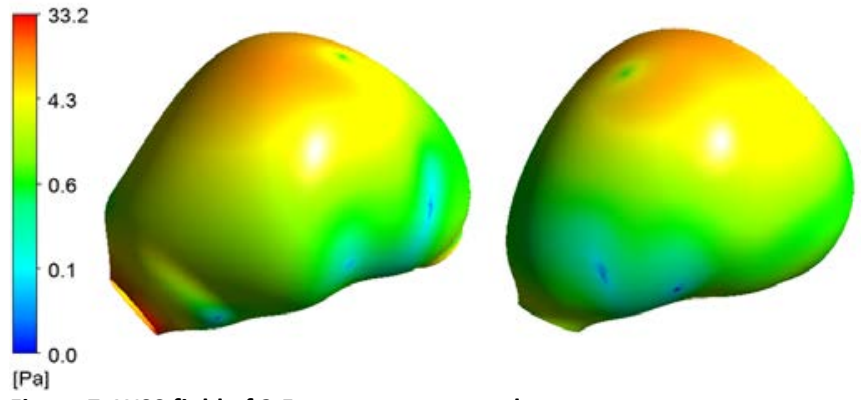

Figure 7. WSS field of 2.5-aneurysm at systole

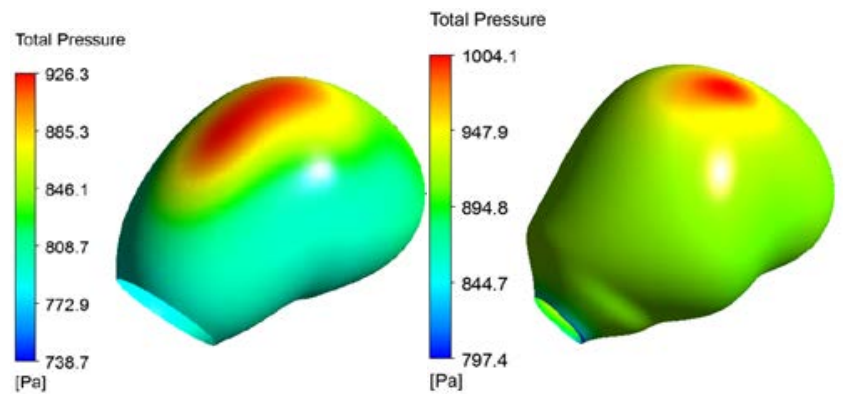

Figure 8. Pressure filed on the wall of 1.2-aneurysm (left) and 2.5aneurysm (right) at systole.

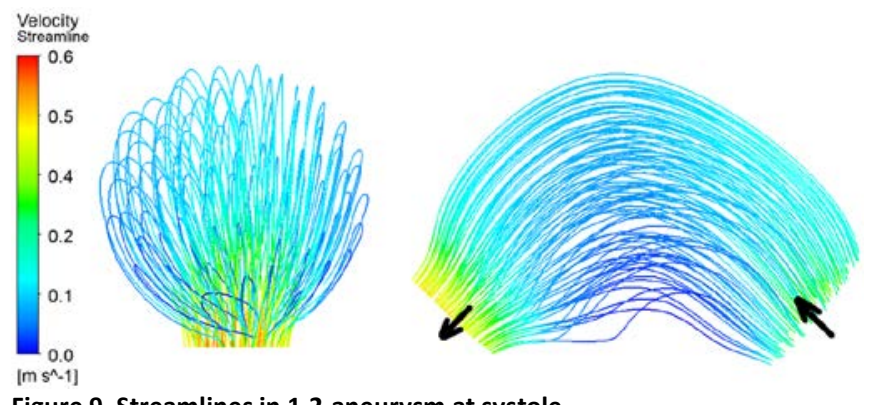

Figure 9. Streamlines in 1.2-aneurysm at systole
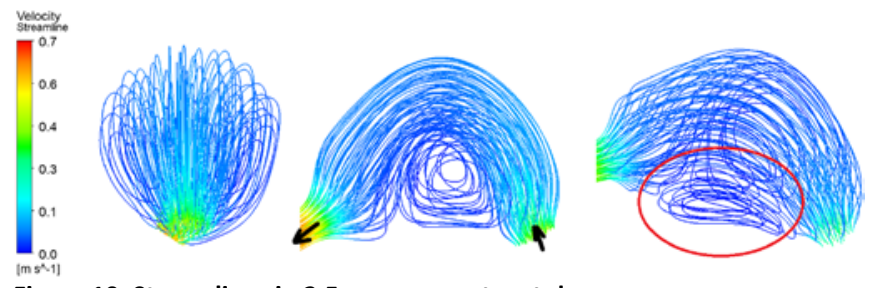

Figure 10. Streamlines in 2.5-aneurysm at systole
Table 4. Morphological factors of two aneurysms

\begin{tabular}{lcccc}
\hline Aneurysms & Size, $\mathrm{mm}$ & Size ratio & Shape & Risk of rupture \\
\hline 1.2-aneurysm & 3.5 & 1.2 & Regular & Low \\
1.6-aneurysm & 4.5 & 1.6 & Regular & Low \\
2.0-aneurysm & 6.0 & 2.0 & Irregular & High \\
2.5-aneurysm & 7.5 & 2.5 & Irregular & High \\
\hline
\end{tabular}

Streamlines in 1.2-aneurysm are shown in Figure 9. Streamlines in 2.5-aneurysm are shown in Figure 10. Black arrows in Figures 9 and 10 indicate the direction of blood flow. Vortex in 2.5-aneurysm is shown on the right in Figure 9 and is marked with red oval. Location of the vortex corresponds to the bleb of the dome of 2.5-aneurysm which is shown in Figure 3.

\section{Discussion}

This article presents the results of biomechanical study of intracranial aneurysms factors of rupture. Literature sources were first studied and factors, as well as their numerical values, were identified, which made it possible to distinguish aneurysms that prone to rupture from aneurysms with small risk of rupture. Then, with the help of numerical modeling, four models of aneurysms were analyzed, as well as the effect of the fracture factors studied on hemodynamics in the dome of aneurysms.

Size of aneurysm is the main characteristic, on which researchers turn their attention. In 1992 [5] authors claimed that size is what distinguishes ruptured aneurysms from unruptured ones. According to Inagawa et al., $4 \mathrm{~mm}$ is the size that characterizes unruptured aneurysms. As criterion for high risk of rupture authors considered aneurysm size of more than $5 \mathrm{~mm}$. The results which were devoted to the problem of searching for criteria for rupturing aneurysms of cerebral vessels were published in Lancet [6]. The study used data from 4,060 patients from 61 medical centers in US, Canada and Europe. The results suggest that asymptomatic aneurysms up to $7 \mathrm{~mm}$ in diameter of circle of Willis anterior arteries have the least risk of rupture.

In most modern studies, relative sizes (size ratio, aspect ratio) of aneurysms are considered to be the criteria of rupture. Nevertheless, in some works [15-18] it was shown that exactly the size of an aneurysm can be a predictor of its rupture. Lv [19] with co-authors considered single small-diameter aneurysms (up to 7 $\mathrm{mm}$ ) of posterior communicating artery, which are not prone to rupture according to ISUIA conclusions. However, more than a half (68 vs 40 ) of small aneurysms of the posterior cerebral artery were ruptured, and their size ratio turned out to be higher, which was confirmed by logistic analysis.

The first work devoted to the study of the aneurysm size ratio was published in 2008 [10]. Size ratio can be fairly simply measured on the basis of 2D angiographic images. In 2009, Tremmel et al. [14] with the help of numerical modeling demonstrated that size ratio is associated with risk of rupture, and high values of this parameter determine aneurysms with blood flows typical to ruptured aneurysms. This conclusion confirms the fact that hemodynamics is one of the causes of aneurysm rupture. Numerical calculations in [14] showed that not the size of the aneurysm, but size ratio determines hemodynamics within the aneurysm. Futami [20] believes that size ratio of 2.1 can be considered as threshold and characterizes the transition of an aneurysm to the stage of high risk of rupture. 
Under the irregular form of an aneurysm, we mean the presence of irregularities and bulges on its wall or "aneurysm in aneurysm" (Figure 1 on the right). This form of aneurysm was described by Weir in 1987 [28]. In recent years, more and more researchers $[4,8,11,16,22,23]$ point to the fact that ruptured aneurysms have irregular shape. From the mechanical point of view, wall of an aneurysm with inhomogeneities can have an inhomogeneous stress field, and structure of the flow inside such aneurysms becomes unstable and inhomogeneous.

Moreover, it was noted that irregular shape of aneurysm dome indicates a high risk of rupture, regardless of its size [11, 22]. The appearance of irregularities on the surface of aneurysm dome indicates degradation of its wall and a possible thrombosis of its lumen [22].

In fact, from the diagnostics point of view, irregular shape is a convenient, accessible and objective criterion, since its detection does not require any calculations or measurements. Irregular shape of an aneurysm can be detected by viewing the results of a computed (CT) or magnetic resonance imaging (MRI) scan.

Authors in [24] stated that low WSS are considered to be dangerous and determine aneurysm rupture. According to the results of Kaneko and co-authors [13], low WSS and vortices in aneurysm dome change the orientation of endothelial cells and promote aneurysm growth and damage to its wall. At the same time, endothelial cells in a normal vessel with laminar blood flow are oriented along the flow and have a regularly oriented structure.

Results in [25] show that low WSS, regardless of other parameters and patient data, are a criterion of multiple intracranial aneurysms rupture. Moreover, ruptured aneurysms, in contrast to unruptured ones, had more complex structure of blood flow and multiple vortices. This can be explained by the fact that as a rule ruptured aneurysms have inhomogeneous structure of the wall, which assumes the presence of aneurysms in aneurysm (so-called irregular shape) $[8,11,22]$.

Numerical modeling of the pulsating blood flow showed that aneurysm grew in regions with low WSS [26]. Results of the numerical simulation were confirmed experimentally. Kojima also showed that blood pressure on the aneurysm wall plays a significant role in the process of aneurysm growth. Larger aneurysms have higher blood pressure in the dome than small aneurysms.

This review showed that a look at predictors of aneurysm rupture has undergone significant changes in recent decades. The absolute aneurysm size was considered earlier. In connection with the development of modern diagnostic methods, as well as the accumulation of a large amount of data on patients with aneurysms, relative dimensions began to be investigated. Moreover, the development of computational methods has made it possible to significantly expand the possibilities of biomechanical modeling and to confirm the fact that hemodynamic factors, in particular, WSS in the dome of aneurysm determine their growth and rupture.

We present analysis of the biomechanical modeling results and show that hemodynamic rupture risk factors work together with morphological. Moreover, their combination allows one to determine aneurysms prone to rupture.

Taking into account morphological factors that can be analyzed on the basis of $3 \mathrm{D}$ aneurysm models presented in this work, two of four considered aneurysms has a high risk of rupture (Table 4) [3, $9,10,11,23,27]$.

Thus, it can be assumed that hemodynamics of the examined aneurysms should be significantly different. Moreover, hemodynamics of 2.0-aneurysm and 2.5-aneurysm should correspond to that for ruptured aneurysms [14].

In fact, simulation results confirmed this assumption. Figure 10 shows presence of vortex in 2.5-aneurysm in the area of bleb of its dome. There were no vortices in 1.2-aneurysm during the entire cardiac cycle (Figure 8). Small insignificant vortex was founded in aneurysm. Moreover, during the entire cardiac cycle average WSS value in 2.5-aneurysm were more than two times lower than the average WSS in 1.2-aneurysm. According to Figure 6, WSS values on most of the dome of 2.0 and 2.5-aneurysm did not exceed 1.5 $\mathrm{Pa}$, which, according to authors [28], is a predictor of rupture.

On the contrary, blood pressure in 2.5-aneurysm was higher than blood pressure in the dome of 1.2-aneurysm during the entire cardiac cycle. WSS distribution in 2.0 and 2.5 -aneurysm was significantly more heterogeneous than in the dome of 1.2- and 1.6-aneurysm. The non-uniformity of WSS distribution, the presence of vortices and complex flow pattern indicate a higher risk of its rupture [25].

Distribution and numerical values of mechanical (hemodynamic) rapture factors suggest that 2.0 and 2.5aneurysms have a higher risk of rupture than 1.2 and 1.6aneurysms. Results of this study indicate that the relationship between aneurysm size and parent vessel diameter and irregular shape of aneurysm are critical to the intra-aneurysmal hemodynamics generated.

It can be concluded that both morphological and hemodynamic factors do not contradict each other, but complement each other.

In this paper, we deliberately modeled aneurysms with rigid walls, so that the mechanical properties of the wall, taken from literature and not corresponding to the individual characteristics of the patient, did not introduce errors into the solution. However, other researchers $[26,29]$ perform calculations with mean values of properties (data from literature), which can both positively and negatively affect the results and their interpretation.

\section{Conclusion}

It was shown that both morphological and hemodynamic factors do not enter into contradiction with each other. This fact suggests that one can develop objective and convenient for use in preoperative planning of surgical treatment methods for assessing risk of rupture of cerebral aneurysms for an individual patient.

\section{Limitations}

The main limitation in this work is the fact that the paper presents only the results of numerical simulations, which at the moment were not compared with experiments on biological samples.

In the future, we hope that we will perform a numerical simulation of the behavior of several aneurysms, for which it is known that they have ruptured or did not ruptured after the diagnosis. This will allow us to compare the pattern of blood flow in aneurysms and other mechanical factors with the diagnosis of this disease. 


\section{Ethical approval}

This article does not contain any studies with human participants or animals performed by any of the authors.

\section{Acknowledgments}

The study was carried out at the expense of a grant from the Russian Science Foundation (project No. 17-71-10191).

\section{Conflict of interest}

There is no any conflict of interest.

\section{References}

1. Cai W, Hu C, Gong J, Lan Q. Anterior communicating artery aneurysm morphology and the risk of rupture. World Neurosurgery 2018; 109: 119-126. https://dx.doi.org/10.1016/j.wneu.2017.09.118.

2. Jiang $H$, Weng $Y X$, Zhu $Y$, Shen J, Pan JW, Zhan RY. Patient and aneurysm characteristics associated with rupture risk of multiple intracranial aneurysms in the anterior circulation system. Acta Neurochir (Wien) 2016; 158(7): 1367-1375. https://dx.doi.org/10.1007/s00701-016-2826-0.

3. Grochowski C, Litak J, Kulesza B, Szmygin P, Ziemianek D, Kamieniak P, et al. Size and location correlations with higher rupture risk of intracranial aneurysms. J Clin Neurosci 2017; 48: 181-184. https://dx.doi.org/10.1016/j.jocn.2017.10.064.

4. Wang GX, Yu JY, Wen L, Zhang L, Mou KJ, Zhang D. Risk Factors for the rupture of middle cerebral artery bifurcation aneurysms using CT angiography. PLoS One 2016; 11(12): e0166654. https://dx.doi.org/10.1371/journal.pone.0166654.

5. Inagawa $\mathrm{T}$, Hada $\mathrm{H}$, Katoh $\mathrm{Y}$. Unruptured intracranial aneurysms in elderly patients. Surg Neurol 1992; 38(5): 364-370. https://doi.org/10.1016/0090-3019(92)90023-G.

6. Wiebers DO, Whisnant JP, Huston J, Meissner I, Brown RD, Piepgras DG, et al. Unruptured intracranial aneurysms: natural history, clinical outcome, and risks of surgical and endovascular treatment. Lancet 2003; 362 (9378): 103-110. https://doi.org/10.1016/S01406736(03)13860-3.

7. Shojima M, Morita A, Nakatomi H, Tominari S. Size is the most important predictor of aneurysm rupture among multiple cerebral aneurysms: post hoc subgroup analysis of unruptured cerebral aneurysm study Japan. Neurosurgery 2018; 82(6): 864-869. https://doi.org/10.1093/neuros/nyx307.

8. Backes D, Vergouwen MD, Velthuis BK, van der Schaaf IC, Bor AS, Algra $A$, Rinkel GJ. Difference in aneurysm characteristics between ruptured and unruptured aneurysms in patients with multiple intracranial aneurysms. Stroke 2014; 45(5): 1299-1303. https://dx.doi.org/10.1161/STROKEAHA.113.004421.

9. Cai W, Shi D, Gong J, Chen G, Qiao F, Dou X, Li H, et al. Are morphologic parameters actually correlated with the rupture status of anterior communicating artery aneurysms? World Neurosurgery 2015; 84(5): 1278-1283. https://dx.doi.org/10.1016/j.wneu.2015.05.060.

10. Dhar S, Tremmel M, Mocco J, Kim M, Yamamoto J, Siddiqui AH, Hopkins LN, Meng $\mathrm{H}$. Morphology parameters for intracranial aneurysm rupture risk assessment. Neurosurgery 2008; 63(2): 185197. https://dx.doi.org/10.1227/01.NEU.0000316847.64140.81.

11. Huang ZQ, Meng ZH, Hou ZJ, Huang SQ, Chen JN, Yu H, et al. Geometric parameter analysis of ruptured and unruptured aneurysms in patients with symmetric bilateral intracranial aneurysms: a multicenter CT angiography study. AJNR Am J Neuroradiol 2016; 37(8): 1413-1417. https://dx.doi.org/10.1016/i.jocn.2017.10.064.

12. Ivanov D, Dol A, Polienko A. Patient-specific hemodynamics and stressstrain state of cerebral aneurysms. Acta Bioeng Biomech 2016; 18(2): 9-17. https://dx.doi.org/10.5277/ABB-00373-2015-03.
13. Kaneko N, Mashiko T, Namba K, Tateshima S, Watanabe E, Kawai K. A patient-specific intracranial aneurysm model with endothelial lining: a novel in vitro approach to bridge the gap between biology and flow dynamics. J Neurointerv Surg 2018; 10(3): 306-309. https://doi.org/10.1136/neurintsurg-2017-013087.

14. 14. Tremmel M, Dhar S, Levy El, Mocco J, Meng H. Influence of intracranial aneurysm-to-parent vessel size ratio on hemodynamics and implication for rupture: results from a virtual experimental study. Neurosurgery 2009; 64(4): 622-630. 10.1227/01.NEU.0000341529.11231.69) (PMID: 19349824).

15. Maslehaty H, Capone C, Frantsev R, Fischer I, Jabbarli R, Cornelius JF, et al. Predictive anatomical factors for rupture in middle cerebral artery mirror bifurcation aneurysms. J Neurosurg 2017; 25: 1-9. https://dx.doi.org/10.3171/2017.2.JNS162705.

16. Mehan WA, Romero JM, Hirsch JA, Sabbag DJ, Gonzalez RG, Heit JJ, Schaefer PW. Unruptured intracranial aneurysms conservatively followed with serial CT angiography: could morphology and growth predict rupture? J Neurointerv Surg 2014; 6(10): 761-766. https://dx.doi.org/10.1136/neurintsurg-2013-010944.

17. Bijlenga $P$, Ebeling $C$, Jaegersberg $M$, Summers $P$, Rogers $A$, Waterworth $A$, et al. Risk of rupture of small anterior communicating artery aneurysms is similar to posterior circulation aneurysms. Stroke 2013; 44: 3018-3026. https://dx.doi.org/10.1161/STROKEAHA.113.001667.

18. Orz $Y$, Alyamany $M$. The impact of size and location on rupture of intracranial aneurysms. Asian J Neurosurg 2015; 10(1): 26-31. https://dx.doi.org/10.4103/1793-5482.144159.

19. Lv N, Feng Z, Wang C, Cao W, Fang Y, Karmonik C, et al. Morphological risk factors for rupture of small $(<7 \mathrm{~mm})$ posterior communicating artery aneurysms. World Neurosurg 2016; 87: 311-315. https://dx.doi.org/10.1016/i.wneu.2015.12.055.

20. Futami K, Nambu I, Kitabayashi T, Sano H, Misaki K, Uchiyama N, Nakada M. Inflow hemodynamics evaluated by using four-dimensional flow magnetic resonance imaging and the size ratio of unruptured cerebral aneurysms. Neuroradiology 2017; 59(4): 411-418. https://dx.doi.org/10.1007/s00234-017-1801-7.

21. Weir B, Amidei C, Kongable G, Findlay JM, Kassell NF, Kelly J, et al. The aspect ratio (dome/neck) of ruptured and unruptured aneurysms. $J$ Neurosurg 2003; 99(3): 447-451. https://dx.doi.org/10.3171/jns.2003.99.3.0447.

22. Lindgren $A E$, Koivisto $T$, Björkman J, von Und Zu Fraunberg M, Helin K, et al. Irregular shape of intracranial aneurysm indicates rupture risk irrespective of size in a population-based cohort. Stroke 2016; 47(5): 1219-1226. https://dx.doi.org/10.1161/STROKEAHA.115.012404.

23. Björkman J, Frösen J, Tähtinen O, Backes D, Huttunen T, Harju J, et al. Irregular shape identifies ruptured intracranial aneurysm in subarachnoid hemorrhage patients with multiple aneurysms. Stroke 2017; 48(7): 19861989. https://dx.doi.org/10.1161/STROKEAHA.117.017147.

24. Cebral JR, Mut F, Weir J, Putman C. Quantitative characterization of the hemodynamic environment in ruptured and unruptured brain aneurysms. AJNR Am J Neuroradiol 2011; 32(1): 145-151. https://dx.doi.org/10.1016/i.wneu.2015.05.060.

25. Jing L, Fan J, Wang Y, Li H, Wang S, Yang X, Zhang Y. Morphologic and hemodynamic analysis in the patients with multiple intracranial aneurysms: ruptured versus unruptured. PLoS One 2015; 10(7): e0132494. https://dx.doi.org/10.1371/journal.pone.0132494.

26. Kojima M, Irie K, Ikeda S, Fukuda T, Arai F, Hirose Y, Negoro M. The hemodynamic study for growth factor evaluation of rupture cerebral aneurysm followed up for five years. Journal of Biomedical Science and Engineering 2012; 5(12A): 884-891. https://dx.doi.org/10.4236/jbise.2012.512A112.

27. Shao $X$, Wang $H$, Wang $Y, X u T$, Huang $Y$, Wang J, et al. The effect of anterior projection of aneurysm dome on the rupture of anterior communicating artery aneurysms compared with posterior projection. Clin Neurol Neurosurg 2016; 143: 99-103. https://dx.doi.org/10.1016/i.clineuro.2016.02.023. 
28. Zhou G, Zhu Y, Yin Y, Su M, Li M. Association of wall shear stress with intracranial aneurysm rupture: systematic review and meta-analysis. Sci Rep 2017; 7(1): 5331. https://dx.doi.org/10.1038/s41598-01705886-w.

29. Cebral JR, Vazquez M, Sforza DM, Houzeaux G, Tateshima S, Scrivano $E$, et al. Analysis of hemodynamics and wall mechanics at sites of cerebral aneurysm rupture. J Neurointerv Surg 2015; 7(7): 530-536. https://dx.doi.org/10.1136/neurintsurg-2014-011247.

\section{Authors:}

Dmitriy V. Ivanov - PhD, Associate Professor, Department of Mathematical Theory of Elasticity and Biomechanics, Saratov State University, Saratov, Russia. http://orcid.org/0000-0003-1640-6091.

Dol V. Alexandr - PhD, Associate Professor, Department of Mathematical Theory of Elasticity and Biomechanics, Saratov State University, Saratov, Russia. http://orcid.org/0000-0001-5842-1615. 